\title{
Sarcoidosis of the aortic root
}

Hrvoje Gasparovic, MD, ${ }^{a}$ Raphael Bueno, MD, ${ }^{b}$ Frank J. Rybicki, MD, PhD, ${ }^{\mathrm{c}}$ Kenneth L. Baughmann, MD, ${ }^{d}$

Robert C. Capodilupo, MD, ${ }^{\mathrm{e}}$ Marzia Leacche, MD, and John G. Byrne, MD, ${ }^{a}$ Boston, Mass, and Bangor, Maine

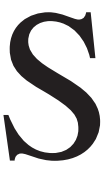

arcoidosis is a clinical entity characterized by noncaseating epithelioid granulomas affecting multiple organs. The cause remains perplexing, but the available evidence points to an exaggerated cellular response as the feature of the disorder. The granulomas, composed of mononuclear phagocytes encircled by CD4 T lymphocytes, have the potential for distorting tissue architecture and thus compromising organ function. Sarcoidosis is a systemic disease, but its symptoms are usually secondary to lung, liver, skin, lymph node, and eye involvement. Clinically manifest cardiac involvement is seen in 5\% of patients. It is characterized by conduction anomalies, pericarditis, and congestive heart failure, with the potential for causing sudden death. We report on an unusual case of cardiac sarcoidosis resulting in aortic valve insufficiency and left ventricular outflow tract (LVOT) obstruction.

\section{Clinical Summary}

A 56-year-old man with a 5-year history of a heart murmur recently had a syncopal episode after newly discovered complete heart block. A transvenous temporary pacing wire was immediately inserted, followed by a permanent atrioventricular sequential pacemaker. Of note, his previous medical history was remarkable for colonic polyps and gastritis. Transthoracic echocardiography revealed a peak gradient across the LVOT of $55 \mathrm{~mm} \mathrm{Hg}$, severe aortic insufficiency (AI), and an ejection fraction of 55\%. Cardiac catheterization showed no obstructive coronary artery disease. The cardiac output was $3.7 \mathrm{~L} / \mathrm{min}$, whereas the pulmonary artery and left ventricular end-diastolic pressures were $49 / 16$ and $30 \mathrm{~mm} \mathrm{Hg}$, respectively. Computed tomography (CT) of the chest showed mediastinal lymphadenopathy. These findings precipitated a lymph node biopsy, which was obtained through a mediastinoscopy. Histologic scrutiny revealed noncaseating granulomas consistent with sarcoidosis. A contrast chest CT with image reconstructions demonstrated a subaortic obstruction $1.7 \mathrm{~cm}$ in length extending into the noncoronary and right coronary cusps (Figure 1). Our surgical strategy consisted of aortic valve replacement

From the Divisions of Cardiac Surgerya and Thoracic Surgery, ${ }^{\mathrm{b}}$ the Department of Radiology, ${ }^{\mathrm{c}}$ and the Cardiovascular Division, ${ }^{\mathrm{d}}$ Brigham and Women's Hospital, Boston, Mass, and Northeast Cardiology, ${ }^{\mathrm{e}}$ Eastern Maine Medical Center, Bangor, Maine.

Received for publication March 12, 2004; accepted for publication March 26, 2004.

Address for reprints: John G. Byrne, MD, Division of Cardiac Surgery, Brigham and Women's Hospital, 75 Francis St, Boston, MA 02115 (E-mail: JBYRNE@PARTNERS.ORG).

J Thorac Cardiovasc Surg 2004;128:761-2

$0022-5223 / \$ 30.00$

Copyright $\odot 2004$ by The American Association for Thoracic Surgery doi:10.1016/j.jtcvs.2004.03.048

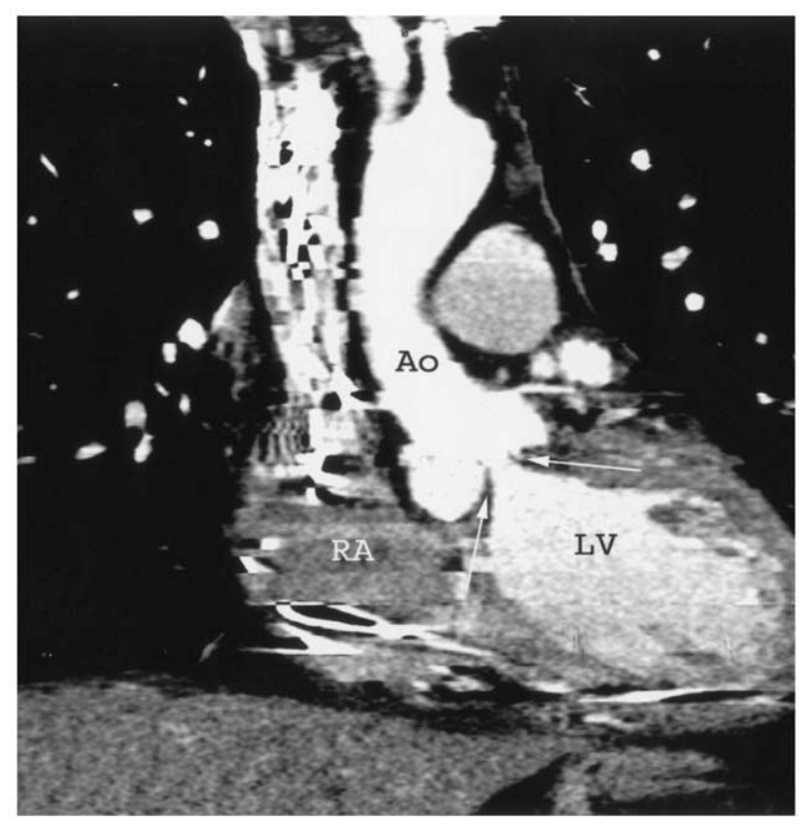

Figure 1. Preoperative coronal CT image reformatted along the LVOT demonstrates subvalvular stenosis (straight arrows). The artifact at the inferior margin of the right atrium (RA) and left ventricle $(L V)$ is due to pacing wires. $A O$, Aorta.

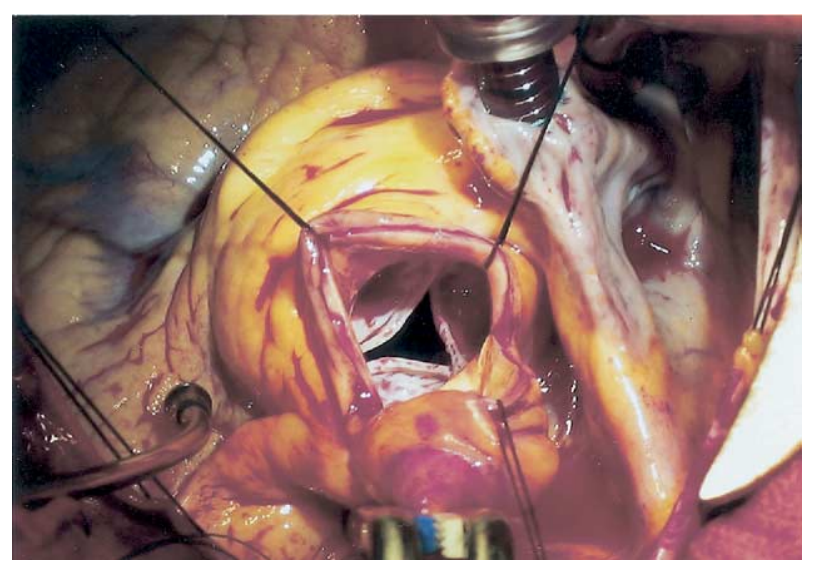

Figure 2. Intraoperative photograph of the aortic root illustrating an infiltrative process responsible for retraction of the aortic valve leaflets with a resulting central defect.

accompanied by relief of the LVOT obstruction. The intraoperative transesophageal echocardiogram corroborated the previously 

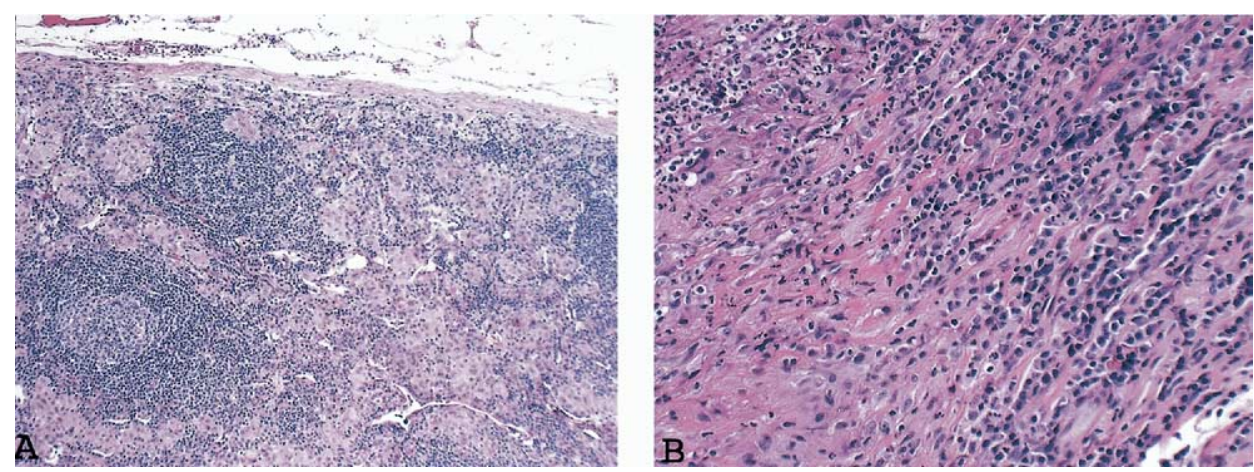

Figure 3. A, Hematoxylin and eosin stain of a lymph node obtained by means of mediastinoscopy, showing noncaseating granulomas. (Original magnification $100 \times$.) B, Hematoxylin and eosin stain of the aortic valve and subvalvular tissue, showing an inflammatory infiltrate of lymphocytes and plasma cells (lower right) within dense fibrous tissue that also contained focally necrotizing granulomatous inflammation (upper left). (Original magnification $200 \times$.)

acquired data, including the subaortic obstruction, severe AI, reduced left ventricular systolic function, and apparent fibrotic infiltration of the LVOT and aortic annulus with radial retraction of the aortic valve leaflets. A median sternotomy was performed, followed by systemic heparinization and institution of cardiopulmonary bypass in the standard fashion. Inspection of the trileaflet aortic valve revealed a fibrotic process that retracted the leaflets in a radial manner, resulting in a central defect, which explained the severe AI. The process was limited to the annulus and the subvalvular area, with no involvement of the sinuses of Valsalva (Figure 2). We proceeded with excision of the aortic valve, followed by resection of the subvalvular obstruction (Figure 3). A 21-mm mechanical valve was then sutured in place. The patient was weaned off cardiopulmonary bypass uneventfully. His hospital stay had been unremarkable, and he was discharged on the fifth postoperative day.

\section{Discussion}

Clinical stigmata of cardiac sarcoidosis are present in 5\% of patients with this unusual inflammatory disease. ${ }^{1}$ The most common presentation is that of an atrioventricular conduction abnormality, followed by arrhythmias, dilated cardiomyopathy, valvular dysfunction, ventricular aneurysm formation, pericarditis, and congestive heart failure. ${ }^{1,2}$ The foundation of the medical management of cardiac sarcoidosis remains corticosteroid therapy, which can result in improvement of myocardial contractility, reduction of left ventricular dimension, and reversal of atrioventricular block. ${ }^{1,2}$ Refractory sarcoidosis unresponsive to escalating steroid and immunosuppression therapy might respond to antibodies directed against tumor necrosis factor $\alpha{ }^{3}$ Endomyocardial biopsy might be useful in providing firm evidence for sarcoidosis. However, because of the patchy distribution of the noncaseating granulomas, biopsy provides a definitive diagnosis in only $22 \%$ to $50 \%$ of cases. ${ }^{1,4}$ Only an abnormal biopsy finding is diagnostic, and treatment should not be delayed in its absence. ${ }^{5}$ One must therefore maintain a high index of clinical suspicion for the diagnosis of cardiac sarcoidosis, even in the absence of convincing myocardial biopsy findings. In the case presented here, the evolution of valvular dysfunction was preceded by complete atrioventricular block. We believe that the case portrayed here represents a unique condition of aortic root sarcoidosis accompanied by involvement of the conduction tissue and further complicated by decrease of the left ventricular function as a result of the same infiltrative cause. The therapeutic strategy for our patient included implantation of a permanent pacemaker, aortic valve replacement, and successful surgical alleviation of his LVOT obstruction, as well as corticosteroid therapy. Rigorous echocardiographic and clinical follow-up is necessary because of the uncertain progression of sarcoidosis involving the LVOT.

We thank Dr Robert Padera of the Department of Pathology, Brigham and Women's Hospital, for his help with providing the histologic images.

\section{References}

1. Syed J, Myers R. Sarcoid heart disease. Can J Cardiol. 2004;20:89-93.

2. Shammas RL, Movahed A. Sarcoidosis of the heart. Clin Cardiol. 1993;16:462-72.

3. Roberts SD, Wilkes DS, Burgett RA, Knox KS. Refractory sarcoidosis responding to infliximab. Chest. 2003;124:2028-31.

4. Sekiguchi M, Yazaki Y, Isobe M, Hiroe M. Cardiac sarcoidosis: diagnostic, prognostic, and therapeutic considerations. Cardiovasc Drugs Ther. 1996;10:495-510.

5. Kato Y, Morimoto S, Uemura A, Hiramitsu S, Ito T, Hishida $\mathrm{H}$. Efficacy of corticosteroids in sarcoidosis presenting with atrioventricular block. Sarcoidosis Vasc Diffuse Lung Dis. 2003;20:133-7. 